\title{
La sobrepoblación: efectos
}

\section{Overpopulation: effects}

\author{
Luis Bouroncle Luna, $\unrhd$ Javier Félix Díaz y Luis Heracles Alcalde \\ Universidad Nacional Mayor de San Marcos. Lima, Perú.
}

Recibido: 25-11-2018 Revisado: 13-12-2018 Aceptado: 14-03-2019 Publicado: 25-05-2019

\section{RESUMEN}

En el presente trabajo, se realiza un análisis de la sobrepoblación, teniendo en cuenta los efectos en todos los ámbitos del desarrollo de la propia sociedad.

Se evalúa la degradación del medioambiente, un aspecto analizado es la urbanización, es decir, el fenómeno de la sobrepoblación de las ciudades.

Otro punto es la emisión del dióxido de carbono y la creación del efecto de invernadero y el consecuente calentamiento global. Un segundo aspecto que se aprecia es la escasez de alimento y agua.

Es evidente que, al incrementar la población, aumenta la demanda de alimentos y el consumo de elementos hídricos. Se termina el artículo haciendo énfasis a un aspecto nunca tomado en cuenta: el de la ética. Se expone un razonamiento ético sobre los problemas del crecimiento de la población y se concluye que el problema de la sobrepoblación es una carencia de educación sobre todas las cosas.

Palabras clave: Efecto invernadero, urbanización, calentamiento global, escasez, contaminación, ética.

\begin{abstract}
In the present work, an analysis of overpopulation is carried out, taking into account the effects in all areas of the development of society itself.

he degradation of the environment is evaluated. For that, one aspect analyzed is urbanization, that is, the phenomenon of overpopulation of cities.

Another point is the emission of carbon dioxide and the creation of the greenhouse effect and the consequent global warming.

A second aspect that is appreciated is the shortage of food and water. It is evident that, by increasing the population, the demand for food and the consumption of water elements develop. The article ends with an emphasis on an aspect never taken into account: ethics of this aspect. An ethical reasoning about the problems of the growth of the population is exposed and it is concluded that the problem of overpopulation is a lack of education on all things.
\end{abstract}

Keywords: Greenhouse effect, urbanization, global warming, scarcity, pollution, ethics. 


\section{INTRODUCCIÓN}

Uno de los problemas globales que afectan al mundo hoy en día es la amenaza de la sobrepoblación.

Se espera que la creciente población de 7 mil millones alcance al menos nueve mil millones, según la proyección media de la ONU. Cada año, la población mundial crece un $1,2 \%$.

Aunque este número puede no sonar alarmante, la población mundial se está duplicando en menos de 50 años.

Cada segundo de cada día, nueve vidas son traídas al mundo. Cada 10 segundos, nacen 44 personas, lo que representa 140 millones de personas por año (Jaramillo et al., 2010).

Al restar esto de la cantidad total de muertes en un año, 84 millones de personas son traídas al mundo con un estimado de 230 mil personas por día.

Eso es el equivalente de otra Alemania o un cuarto de los Estados Unidos.

Por lo tanto, no hay duda de que el planeta se está superpoblando. En el libro ¿Está Ileno el planeta?, Ian Goldin escribe que en el planeta que contiene más de 7 mil millones de personas, el $60 \%$ vive en Asia, el $15 \%$ en África, el $11 \%$ Europa, el $9 \%$ en América Latina y el Caribe, $5 \%$ en América del Norte y menos del $1 \%$ en Oceanía.

En Singapur, más de 7 mil personas viven por kilómetro cuadrado, mientras que, en Australia, en promedio, tres personas habitan en la misma área (Jaramillo et al., 2010). Los activistas ambientales reconocen el problema, mientras que el resto piensa que podrán superar esta crisis, ya que pudieron sobrevivir a eventos ambientales similares en el pasado, pero no se dan cuenta de que la naturaleza dio un giro irreversible y que lo que ocurre no puede volver a como era antes.

Muchas personas tienden a centrarse en los objetivos a corto plazo para enriquecerse y experimentar placer en lugar de prestar toda su atención a la protección del medio ambiente para los objetivos a largo plazo.

Es de suma importancia que los humanos comprendan que nuestro planeta es un sistema limitado y que ninguna ayuda externa vendrá para ayudar a la escasez de recursos naturales que los humanos pronto experimentarán.

Por lo tanto, el objetivo de este artículo es concientizar sobre el impacto de la sobrepoblación en el medio ambiente desde el punto de vista de la ética.

\section{POBLACIÓN Y CAMBIO CLIMÁTICO: DEGRADACIÓN SOBRE EL MEDIO AMBIENTE}

\subsection{Urbanización}

En comparación con las áreas rurales, la tasa de fertilidad en las áreas urbanas es baja debido a una amplia gama de oportunidades ofrecidas a las mujeres.

Más oportunidades económicas, un mejor acceso a la educación, programas expansivos de planificación familiar y atención médica adecuada tienen un impacto en las decisiones de las mujeres sobre la reproducción. 
Por ejemplo, las tasas de fertilidad urbana en Kenia, Tanzania y Uganda son alrededor de 40 \% más bajas que las tasas rurales y muy por debajo de los promedios nacionales. Sin embargo, la migración desde el campo o la expansión de las aldeas hacia ciudades son factores que contribuyen al crecimiento urbano.

Según las estadísticas, 6,500 millones de personas vivirán en ciudades para el 2050, más que la población total del mundo hoy en día (Sánchez, 2008).

La urbanización excesiva tiene un impacto devastador en el medio ambiente.

Las ciudades crean el 80 por ciento de los gases de efecto invernadero. Esto se explica por el efecto de isla de calor urbano bajo el cual los entornos rurales tienen una temperatura más baja del aire y la superficie que las áreas urbanas.

Los principales factores causales del efecto son la arquitectura de la ciudad, especialmente los edificios de gran altura que atrapan el calor, el tamaño del área urbana y el terreno cubierto con superficies impermeables al agua, como edificios y carreteras.

El proceso de urbanización permitió la contaminación de los recursos hídricos, la biodiversidad, el aire y el funcionamiento de los ecosistemas.

Los recursos hídricos, como ríos, deltas y costas, son las características principales que los urbanistas buscaban cuando construían y expandían ciudades.

Sin embargo, después de la urbanización, las fuentes acuáticas se han modificado para satisfacer las necesidades de las ciudades, lo que supuso un costo para estos ecosistemas locales que demostró ser una protección contra desastres naturales.

En India y Sri Lanka, por ejemplo, los bosques de manglares agotados dejaron a las comunidades costeras vulnerables al tsunami de 2005, mientras que a las comunidades donde los ecosistemas permanecieron intactos les fue mucho mejor (Thompson y Stoutemyer, 1991).

Además, otra consecuencia de la urbanización es la eutrofización, o un aumento de nutrientes químicos en los recursos hídricos, que se produce debido a la producción urbana de dióxido de carbono y otros gases de efecto invernadero y contaminantes que limpian las superficies impermeables en los recursos hídricos.

El proceso finalmente contamina la calidad del agua y afecta los ecosistemas marinos. Esto es una consecuencia de los países en desarrollo que experimentan una rápida urbanización sin implementar protecciones ambientales e identificar químicos modernos que deterioren la infraestructura.

En esta etapa, los países en desarrollo contaminan el medio ambiente, ya que no implementan la construcción de instalaciones para tratar los desechos peligrosos.

En esas ciudades, las infraestructuras de aguas pluviales a menudo no separan la escorrentía de las descargas de aguas residuales, lo que crea problemas agudos de contaminación en las vías navegables de los receptores (George et al., 2014). 
El impacto de la actividad humana es devastador para la biodiversidad.

El crecimiento de las ciudades empuja a los animales fuera de sus hábitats que se están urbanizando. En el proceso de migración masiva a las ciudades y la falta de programas de planificación familiar, se lleva a cabo una extinción masiva de especies.

Los científicos de EE.UU. Dicen que la Tierra se encuentra en medio de su sexta extinción masiva de plantas y animales, con casi el $50 \%$ de todas las especies desapareciendo, mientras que se estima que la mitad de los mamíferos del mundo estaban disminuyendo en población y más de un tercio probablemente se enfrentan a la extinción.

El mayor número de especies eliminadas se encuentra en primates y mamíferos marinos. Aunque la mayoría de los ecosistemas en la Tierra se han visto afectados por la urbanización, la migración a las áreas rurales tiene un impacto peor, a medida que se extienden mayores superficies de tierra y se explotan más recursos (Suárez et al., 1992).

La actividad humana es, por lo tanto, la única causa de extinción. En la actualidad, los pueblosyciudades seextienden, seconstruyen caminos a través de ecosistemas frágiles, se perfora petróleo y gas y se expanden las actividades mineras destructivas.

Se reducen los bosques para la tala, los lagos, mares y ríos se contaminan, los arrecifes de coral se degradan y se fragmenta el desierto. Y se bombea toneladas y toneladas de gases de efecto invernadero a la atmósfera (Tracy y Oskamp, 1984).

Además de la contaminación de los recursos hídricos y la degradación de la biodiversidad, la calidad del aire también se afecta. La cantidad de contaminación liberada a la atmósfera de las fábricas, los hogares y los automóviles es mucho mayor de lo que el medio ambiente puede soportar.

Esto se refiere particularmente a los países en desarrollo, como las áreas urbanas de los países asiáticos donde la gasolina con plomo todavía se usa ampliamente, en comparación con los países desarrollados que lograron reducir sus emisiones de plomo en la industria automotriz.

Además, es probable que las emisiones de dióxido de azufre, partículas y plomo en los automóviles sean significativamente mayores en el futuro debido al aumento de la propiedad de automóviles en muchas ciudades, gracias a la introducción de automóviles baratos.

Por ejemplo, en China, las ciudades que emiten muchas más emisiones que sus contrapartes rurales.

Los residentes de áreas urbanas, como Beijing y Shanghái, no son responsables de la cantidad de emisiones que emiten estas ciudades.

Las áreas urbanas chinas son importantes productores de bienes para los países occidentales y, por lo tanto, sus fábricas contribuyen en gran parte a las emisiones globales de gases de efecto invernadero.

\subsection{Dióxido de carbono y efecto de invernadero}

Una huella de la humanidad en el medio ambiente global es evidente en el aumento del dióxido de carbono y la disminución del ozono en la atmósfera.

La quema de madera, carbón y petróleo, junto con las erupciones volcánicas y el 
proceso de respiración de animales, plantas y microorganismos, son compuestos que producen dióxido de carbono.

En los últimos 200 años, el dióxido de carbono ha aumentado exponencialmente $y$, en este período, aumentó en un cuarto en la atmósfera.

Sin embargo, hasta que los humanos han comenzado a quemar combustibles fósiles y cambiar el ritmo de producción de dióxido de carbono, el proceso natural de liberación de dióxido de carbono a la atmósfera ha sido equilibrado.

Cuando las plantas mueren, el dióxido de carbono que absorben se queda en el suelo y luego sirve para formar reservas de petróleo, gas y carbón debajo de la tierra.

El dióxido de carbono absorbido en los océanos es utilizado por las plantas microscópicas fotosintéticas para producir compuestos que contienen carbono que forman sus células.

El carbono se hunde hasta el fondo del océano y permanece enterrado.

Mientras tanto, los volcanes han estado muy activos a lo largo de la historia y, con el tiempo, han estado liberando dióxido de carbono a la atmósfera, pero las plantas absorben estas cantidades excesivas de dióxido de carbono para la fotosíntesis y se depositan en el suelo, formando un equilibrio natural.

Proceso de emisión y absorción de dióxido de carbono.

Con el inicio de la revolución industrial, los humanos comenzaron a depender en gran medida de la energía producida por la quema de combustibles fósiles.
Como los bosques son una fuente abundante de carbono, la deforestación permitió a los humanos dejar su rastro en la contaminación ambiental.

La pérdida de la cubierta forestal ha aumentado significativamente en los últimos cientos de años, pero las selvas tropicales de Brasil, Indonesia y Zaire se encuentran bajo un foco especial ya que estas selvas están siendo destruidas a una velocidad de 100 acres por minuto, o 52,6 millones de acres al año. Inevitablemente, por lo tanto, la deforestación está estrechamente relacionada con un aumento de la población.

Con el crecimiento de la población, los bosques se reducen y los árboles se talan o se queman para satisfacer las crecientes demandas de las personas, especialmente en los países en desarrollo que dependen de la leña como su única fuente de combustible.

Las estadísticas proporcionadas por la Organización de las Naciones Unidas para la Agricultura y la Alimentación (FAO) muestran que en 1989 más del 70 \% de las familias en el Tercer Mundo usaban leña para combustible.

Por lo tanto, la hambruna en África tiene su origen en la erosión y la degradación del suelo que resulta de la deforestación en la búsqueda de leña.

En el pasado, las personas han tratado de usar la leña de manera sostenible, de modo que en el futuro la naturaleza no se enfrenta a una escasez de madera.

Sin embargo, hoy en día, la leña se utiliza a un ritmo mayor y en algunas partes del mundo, los bosques casi se cortan en el suelo. 
La creciente población de India y Nepal utiliza la leña para cocinar y calentar, dejando el fondo de las montañas del Himalaya completamente deforestado, lo que dificulta la agricultura debido a las inundaciones.

La capa superficial del suelo se lava y el bosque necesita un período de tiempo más largo del que los humanos permiten renovarse.

Además, la deforestación contribuye a la formación de otros problemas ambientales, como el calentamiento global, la erosión y la desertificación.

La deforestación conduce a la combustión de carbono y libera dióxido de carbono a la atmósfera.

Los árboles ya no protegen la capa superficial del suelo absorbiendo la lluvia durante la vegetación y almacenando el agua en las capas de la capa superior del suelo, sino que la lluvia se convierte en manantiales y arroyos, lo que causa erosión e inundaciones.

La extinción de especies es otro resultado de la deforestación, ya que cada organismo vivo se adapta a su hábitat natural, que cuando se destruye amenaza la supervivencia del organismo.

A pesar de que todos los hábitats naturales están experimentando crisis en la actualidad, las selvas tropicales están en particular peligro porque poseen la mayoría de las especies vivas del mundo que se destruyen debido a la deforestación.

Según el biólogo Edward O. Wilson de la Universidad de Harvard, cada año se desvanecen de 10,000 a 17,500 especies de plantas, aves, insectos, reptiles y mamíferos Wilson (Wilson, 1978).

Los científicos predicen que la actividad humana de la deforestación tendrá un impacto duradero en el medio ambiente.

El final del siglo XIX y principios del siglo XX mostró que la deforestación global aumentó significativamente la concentración de dióxido de carbono en la atmósfera debido a la quema de combustibles fósiles, lo que representa un aumento de más de tres veces las emisiones de $\mathrm{CO}_{2}$.

Dado que el petróleo, el carbón y el gas natural se queman a una tasa mayor que antes, existe una fuerte correlación entre el uso de energía global y el dióxido de carbono en la atmósfera.

La quema de combustibles fósiles libera dióxido de carbono a una tasa de 6 millones de billones de toneladas de carbono por año, y estas partículas permanecerán en la atmósfera durante al menos 100 años.

Se requieren décadas o siglos para que los procesos globales alcancen el equilibrio (es decir, para volver a un estado estable).

La cantidad de dióxido de carbono en la atmósfera es inferior al uno por ciento, sin embargo tiene un impacto en la temperatura global.

En la década de 1890, un ganador del Premio Nobel sueco, Scante Arrhenius fue el primero en observar el efecto del dióxido de carbono en la temperatura.

Sus observaciones introdujeron el "efecto invernadero", o un aumento en la temperatura de la Tierra que se calienta 
como un invernadero que atrapa el calor solar.

El proceso de efecto invernadero comienza cuando el dióxido de carbono atrapa la energía térmica en la atmósfera de la Tierra.

La Tierra es calentada por el sol y emite energía de vuelta al espacio, pero parte de la energía queda atrapada antes de que alcance el espacio, y la temperatura de la atmósfera aumenta.

La cantidad de energía atrapada es proporcional a un aumento de las concentraciones de dióxido de carbono, que da forma a la temperatura atmosférica.

A pesar de que China es el mayor contaminante, Estados Unidos ocupa el segundo lugar ya que su economía se basa en los combustibles fósiles.

Aunque Estados Unidos tiene el 4,5\% de la población mundial, produjo el $18 \%$ de las emisiones de dióxido de carbono del mundo en 2010, según la Administración de Información de Energía de los Estados Unidos.

Los científicos y expertos en cambio climático estimaron que el umbral más alto aceptable de dióxido de carbono en la atmósfera equivale a 350 partes por millón, y si la cantidad supera la estimación sugerida, la Tierra experimentará graves consecuencias del calentamiento global. Hoy en día, el planeta tiene 392 partes por millón de $\mathrm{CO}_{2}$.

\section{CALENTAMIENTO GLOBAL}

A pesar del amplio debate y la controversia sobre el calentamiento global como una amenaza potencial para nuestro medio ambiente, los expertos proporcionan estadísticas para demostrar que la Tierra está en peligro.

En comparación con el comienzo del siglo $X X$, las temperaturas globales han aumentado en 1 grado Fahrenheit $y$ continúan aumentando.

En 1920, el Parque Nacional Glacier de Montana tenía 150 glaciares, pero hoy contiene menos de 30 .

Las nieves del monte Kilimanjaro en Tanzania se han derretido en un $80 \%$ desde 1912, y muchos afirman que estas nieves simbólicas podrían desaparecer para 2020. Los nevados del Huascarán en Perú están corriendo la misma suerte.

Al mismo tiempo, la quema de combustibles fósiles dio como resultado un aumento de las emisiones de carbono para casi el doble de la tasa de población.

En la atmósfera, el dióxido de carbono aumentó un $30 \%$ sobre los niveles industriales.

La quema de combustibles fósiles y la fuerte actividad económica dieron como resultado un aumento de las emisiones de carbono en áreas urbanas altamente desarrolladas.

Se proyecta que las emisiones en China crecerán más de tres veces más rápido que la población en los próximos 50 años, debido al auge de la economía que depende en gran medida del carbón y otras fuentes de energía ricas en carbono.

Los científicos predicen que en los próximos 500 años los países en desarrollo cuadruplicarán las emisiones de carbono, mientras que las naciones desarrolladas aumentarán la contribución del carbono en un $30 \%$. 
La consecuencia de un aumento de las emisiones de carbono en la atmósfera tiene un impacto devastador en el cambio climático.

Todos los años, los bosques en California sufren incendios severos, mientras que las inundaciones destruyen las aldeas costeras en Bangladesh.

Las capas de hielo en el Ártico se están derritiendo a un ritmo acelerado y los osos polares se están ahogando, mientras que las ranas se están convirtiendo en especies extintas en la selva tropical de Costa Rica.

En el informe sobre el calentamiento global, el Panel Intergubernamental sobre el Cambio Climático de las Naciones Unidas (IPCC) predijo en 2007 que el cambio climático tendrá un efecto desastroso sobre la naturaleza.

Para 2080, según los científicos, la cantidad de personas que pasarán hambre en el mundo podría aumentar entre 140 millones y 1 mil millones, dependiendo de la cantidad de gases de efecto invernadero emitidos en los próximos años.

Además, es mucho más probable que ocurran enfermedades como la malaria, la fiebre, las muertes relacionadas con el calor y otras enfermedades. Para 2020, aproximadamente 250 millones de personas que viven en países en desarrollo podrían enfrentar escasez de agua. Si las temperaturas promedio aumentan de 2,5 a $4,5{ }^{\circ} \mathrm{F}$, aproximadamente el 20 a $50 \%$ de las especies de plantas y animales enfrentan un mayor riesgo de extinción.

No hay duda de que los más pobres de la población mundial serán los más afectados, pero incluso los países con recursos poderosos, como Estados Unidos, sufrirán el impacto del cambio climático.
Vinculado a esto, en las próximas décadas, se pronostica que la demanda de energía se duplicará y las naciones ricas usarán tanta energía como su población crecerá.

Si el consumo de energía per cápita es alto, el crecimiento de la población no necesita aumentar significativamente para que la energía se use en exceso, ya que la demanda total de energía aumentará por sí sola.

En los Estados Unidos, por ejemplo, los 75 millones de personas que se prevé que se sumarán a la población para 2050 aumentarán la demanda de energía.

La producción de petróleo en todo el mundo alcanzó su punto máximo en 1979 y se prevé que alcance su punto máximo nuevamente en 2025, lo que afectará los niveles de precios, ya que el petróleo sigue siendo el único combustible dominante en el mundo.

En un futuro cercano, los factores del cambio climático se originarán en países con la actividad económica más alta, como Asia, donde se espera que el consumo crezca $361 \%$, aunque la población crecerá solo un $50 \%$.

También se espera que el consumo de energía aumente en América Latina (340 \%) y en África (326 \%).

\section{CÓMO SE PUEDE ALIMENTAR A UNA POBLACIÓN DE NUEVE MIL MILLONES}

\subsection{Escasez de alimentos}

La relación entre la cantidad de personas en el planeta y la cantidad de alimentos es directa.

Cada individuo necesita una ingesta diaria de dos a tres mil calorías, donde la dieta está equilibrada y llena de nutrientes necesarios. 
A medida que crece el número de personas, la demanda de alimentos en última instancia aumenta.

Por lo tanto, según el biólogo Paul Ehrlich, la carrera entre la población y la comida nunca se puede ganar.

Aunque las Naciones Unidas afirman que el mundo produce suficiente grano para alimentar a los humanos con 3,500 calorías por día, las estadísticas demuestran ser diferentes.

La cosecha mundial de cereales en 2010 fue la tercera más alta de la historia, pero 950 millones de personas padecían hambre desesperada y más de mil millones más no podían obtener suficientes nutrientes para mantener una buena salud.

Aún más impactante, el 60 \% de las personas hambrientas del mundo son pequeños agricultores y el $20 \%$ son trabajadores agrícolas sin tierra.

En el período comprendido entre 1950 y 1984 , la producción mundial de granos superó el tamaño de la población, pero después de 1984 el mundo experimentó un crecimiento más lento a medida que la cosecha de granos disminuyó por persona en un $7 \%$ o un $0,5 \%$ al año.

La falta de área de cultivo y la caída de agua de riego por persona, así como el uso no efectivo de fertilizantes, son factores que contribuyen a una gran brecha alimentaria.

A mediados del siglo, las tierras de cultivo aumentaron un $19 \%$, pero la población creció globalmente en un $132 \%$.

Esto ha llevado a los países en desarrollo a perder la capacidad de alimentarse, y el crecimiento de la población logró eliminar las tierras de cultivo de la producción.

Sin embargo, la desigualdad en la producción y distribución de alimentos es otro factor que contribuye a la brecha alimentaria mundial.

Las corporaciones agrícolas gigantes operan para maximizar sus ganancias al colocar los alimentos en áreas donde las personas tienen capacidad financiera para comprarlos.

Por lo tanto, la disponibilidad diaria de alimentos es de aproximadamente 4,000 calorías por persona en el Norte del planeta, pero solo 2,500 calorías en el Sur, incluida la distribución desigual dentro de estos países.

Por otro lado, en Canadá, se desperdicia el 40 $\%$ de todos los alimentos producidos.

Los consumidores desperdician alrededor del $20 \%$ de los alimentos, mientras que el resto se destina a la cosecha, el transporte, el embalaje, los restaurantes y las tiendas, los estudios muestran otros ejemplos de desigualdad alimentaria, donde aproximadamente el cuarenta por ciento del grano cosechado se convierte en carne y combustible.

En lugar de alimentarse, 38 humanos alimentan a los animales y envían el maíz a las granjas industriales, donde el ganado las sustituye por pasto.

No obstante, este parece ser un método inútil para el uso de granos porque una hamburguesa de media libra que se consume diariamente por un consumidor en Brasil o en los Estados Unidos consume suficiente grano para satisfacer el total diario de energía y proteínas que necesitan las tres personas en la India con una dieta combinada de granos y leche. 
De manera similar, en lugar de alimentar a la creciente población de los países económicamente pobres, las naciones ricas aprovecharon el maíz para quemarlo en un biocombustible que activará los motores de los automóviles de sus consumidores más ricos.

Por lo tanto, entre 2002 y 2008 los precios mundiales de los alimentos aumentaron a más del doble. En la investigación llevada a cabo por el Banco Mundial, se concluyó que la principal razón para el aumento de los precios fue el aumento de la producción de biocombustibles en los Estados Unidos y entre las naciones de la Unión Europea.

En 2007, los vehículos estadounidenses quemaron suficiente maíz para cubrir todas las necesidades de importación de los ochenta y dos países más pobres.

Dos años más tarde, los fabricantes de etanol en los Estados Unidos produjeron más biocombustibles que la producción combinada de granos de Canadá y Australia.

Mientras que las naciones desarrolladas están valorando más sus ganancias y proponen programas para los métodos de cultivo modernos, un gran porcentaje de personas en el mundo nunca han visto carne, leche, pescado y huevos en su comida diaria.

Las grandes plantaciones de café en Brasil ocupan una gran cantidad de tierra para cultivar un cultivo que tiene poco valor nutricional.

Como el café es un cultivo rentable, Brasil lo exporta a América del Norte y Europa en lugar de centrarse en los problemas domésticos del hambre.
Por lo tanto, la falta de nutrientes necesarios, como los aminoácidos que forman proteínas, produce inanición y energía inadecuada, lo que conduce a enfermedades causadas por deficiencias de proteínas.

\subsection{Escasez de agua}

El crecimiento de la población mundial resultó en la presión sobre el agua, el único recurso valioso que tiene una tasa de suministro finita.

En la última mitad del siglo, los humanos usaron la mitad del recurso, lo que significa que una cantidad estricta permanecerá disponible en la próxima mitad del siglo.

El agua ya no estará disponible para usos indirectos, como regar los campos de golf o llenar piscinas, y muchas especies pueden extinguirse en áreas severamente secas, como Phoenix o Las Vegas.

El impacto será terrible en los países en desarrollo que dependen del agua para los cultivos para combatir la desnutrición y la inanición persistentes.

Sin embargo, la escasez de agua ya se experimenta en todo el mundo, ya que más de un tercio de todas las personas en la Tierra viven en áreas donde el agua escasea, y 1.700 millones de personas residen en regiones donde la escasez crónica de agua dificulta la producción de cultivos y el desarrollo económico.

Lima se encuentra en una zona de "escasez económica del agua", mientras que el Perú entero se divide en esta posición y la de "escasez física del agua", en parte al llamado "estrés hídrico" que se produce por el crecimiento demográfico y económico de la región, con implicancia de un mayor consumo de agua potable y contaminación de las fuentes primarias. 
En el Perú se consume en promedio poco más de 150 litros de agua por habitante cada día.

La media del consumo de nuestro país es similar al de naciones como Noruega, Finlandia, Reino Unido o Alemania, donde sí tienen agua suficiente para ofrecer a su población y no padecen de carencias hídricas.

En África, las mujeres caminan por millas todos los días y llevan jarras de agua. Aproximadamente mil millones de personas todavía no tienen acceso al agua potable en la actualidad.

La demanda de agua aumenta más rápido que la propia población.

Entre 1950 y 1980, el uso mundial del agua se triplicó con creces; en los Estados Unidos aumentó en un $150 \%$ durante ese período de 30 años, aunque la población del país creció solo en un $50 \%$.

En 1990, cada día una familia estadounidense de cuatro personas usaba 243 galones de agua, lo que a nivel nacional equivalía a 400 mil millones de galones de agua por día.

Existen tres formas fundamentales en que las personas usan el agua.

La mayoría de las creencias se basan en el argumento de que el uso doméstico, que implica beber, cocinar, bañarse, lavarse e higienizarse, es el mayor factor de insuficiencia.

Otros sostienen que las industrias utilizan el agua más allá de un nivel aceptable para impulsar las plantas y enfriar o limpiar los procesos en ellas, pero el factor más importante de todos es el uso del agua en la agricultura para el riego, que representa el
$73 \%$ de todo el uso de agua dulce en todo el mundo y por más del 80 \% del uso del agua en los Estados Unidos.

El riego es un proceso muy sensible en el que se necesita una cantidad adecuada de agua para combatir la salinización del suelo, pero al mismo tiempo para evitar que los fertilizantes y pesticidas se viertan en los hábitats acuáticos.

La consecuencia de la escasez de agua es evidente en el drenaje de los ríos Nilo, Amarillo y Colorado, que casi no tienen agua para derramar en el mar. No obstante, en los Estados Unidos, el gobierno construyó represas que abastecerían de agua a los agricultores en el Oeste a un precio bajo su costo real.

El agua está contaminada y contaminada con desechos humanos, que forman enfermedades bacterianas, como el cólera, la poliomielitis y la hepatitis infecciosa, pero también contiene parásitos y sustancias químicas tóxicas que conducen al cáncer y enfermedades neurológicas.

El hecho de que el crecimiento de la población exceda la cantidad total de agua disponible demuestra el lento proceso de reposición del agua en las fuentes de agua subterránea, o acuíferos, que casi se consideran fuentes no renovables.

Los embalses subterráneos han estado llenándose durante más de mil años por el ciclo de lluvia, pero hoy en día la mayoría de ellos están vacíos y contaminados.

Un ejemplo del aumento de la demanda de pozos es la Franja de Gaza, ubicada entre Israel y Egipto, donde el acuífero se ha vaciado a medias. 
A medida que se agotaba el agua dulce, el agua salada del mar Mediterráneo entró en el pozo y lo dejó contaminado.

Los humanos no solo están eliminando excesivamente las fuentes naturales necesarias para su supervivencia, sino que también están destruyendo los hábitats de los animales.

\section{UN RAZONAMIENTO ÉTICO SOBRE LOS PROBLEMAS DEL CRECIMIENTO DE LA POBLACIÓN}

Desde que el homo sapiens emprendió a evolucionar su actividad adaptativa y de supervivencia, gracias a su capacidad de producir para sus necesidades, lo que dio lugar a la actividad económica, que se convirtió en una nueva fuerza reguladora primaria del crecimiento de su población, aparecieron nuevas regularidades.

Esta actividad creativa del hombre hizo posible la aparición de la cultura como un conjunto material y espiritual de su creación, como un nuevo subsistema que se integró en la naturaleza y brindó nuevas regularidades en el crecimiento de su reproducción, de manera contextualizada, para los diferentes grupos dentro de esta especie.

La capacidad de proteger a sus descendientes, con nuevas acciones inteligentes 0 aprendidas, que añadieron eficiencia a los comportamientos biológicos heredados, así como a la transformación de la actividad sexual, de los medios reproductivos gobernados por ciclos hormonales, en medio del placer regido por presiones socioeconómicas, morales, religiosas y psicológicos, entre otras actividades, transformaron y diferenciaron radicalmente los determinantes del crecimiento de la población humana.
Los eventos mencionados contribuyeron a transformar el ritmo natural del crecimiento de la población humana en un fenómeno exponencial, sujeto fundamentalmente a regularidades económicas, sociales y psicológicas que, por más arbitrarias que parezcan, siempre expresan una necesidad, un sentido social y personal, que debe ser estudiada, en cada comunidad concreta.

Desde finales del siglo $\mathrm{XIX}$, prestigiosos médicos y economistas (Virchow, Marx, Percival) han registrado la relación entre el desempleo, la marginación, la miseria y la sobrepoblación, pero las posibilidades que ofrece el desarrollo económico y tecnológico les hicieron olvidar estas verdades.

Estas verdades y la realidad no pueden ser ignoradas por especialistas experimentados que las estudiaron y reaparecieron con fuerza en el trabajo Equidad y Transformación Productiva de la Comisión de Economía para América Latina, donde se precisa que los países más pobres tienen las tasas más altas de crecimiento de la población.

A su vez, los niveles de fertilidad de los sectores en situación de pobreza en los países de la región son más altos que los de los otros estratos.

Los problemas que tienen su origen en regularidades económicas o actividades sistemáticas y habituales del modo de vida, son movidos por fuerzas generalmente oscuras para la conciencia, y si se unen a fuerzas provenientes de intereses ideológicos, su probabilidad de solución se ve obstruida y se convierte en un problema desconcertante Esta situación ha afectado la solución a los problemas de la reproducción humana y el crecimiento de la población con un mayor daño en las comunidades y en las personas 
menos favorecidas por el desarrollo cultural y la personalidad.

Antes del estudio de los problemas generados por el crecimiento de la población en este momento, no podemos partir de la muy citada teoría de Malthus o de otros enfoques con más o menos aceptación, sino que fueron concebidos cuando las conquistas de la ciencia, la tecnología y la ética, no había ofrecido tantas posibilidades para comprender y luchar contra esos problemas.

Por todos esos motivos, la concepción del mundo de la población y sus criterios acerca de la familia y su reproducción, deben ser educados éticamente.

Vivir éticamente es pensar en cosas y situaciones que están más allá de nuestros propios intereses. Cuando se piensa éticamente se deviene en un único ser, con necesidades y deseos propios, sin duda, pero que vive entre otros seres que también tienen necesidades y deseos.

Cuando actuamos éticamente, deberíamos ser capaces de justificar lo que hacemos y esta justificación debe ser tal que pueda convencer a cualquier ser razonable.

\section{CONCLUSIONES}

La urbanización excesiva tiene un impacto devastador en el medio ambiente; una consecuencia de la sobrepoblación en el medio ambiente global es evidente en el aumento del dióxido de carbono y la disminución del ozono en la atmósfera; otra consecuencia es el calentamiento global, la destrucción de las reservas de hielo y el devastador cambio climático.

La falta de alimentos requiere de una multiplicidad de acciones.
Los alimentos no son como cualquier otro bien, no puede ser objeto de especulación: la alimentación y la producción de biocombustibles no pueden entrar en competición.

Los estados deben asumir su responsabilidad y diseñar políticas que modifiquen el rumbo actual, así mismo la escasez de agua y su contaminación es otra grave consecuencia de la sobrepoblación.

Los problemas del crecimiento de la población en sociedades menos favorecidas, son un problema muy delicado.

Se puede tratar de varias maneras, como, por ejemplo, con el desarrollo de los medios y políticas para la planificación familiar y el control de natalidad.

Llevar una vida con principios éticos sería, no solo vivir por nuestros intereses personales y con la creencia de que llevar una buena vida, depende de la acumulación de bienes y riquezas materiales, si no vivir tomando decisiones correctas para el bien de la sociedad en conjunto.

\section{REFERENCIAS BIBLIOGRÁFICAS}

Arenas Puma, A. L. (2017). Planificación y nivel de ejecución presupuestal y financiero de los proyectos de inversión de la sede central del Gobierno Regional de Arequipa 2016. Repositorio de la UNAS.

Becker, D. (1997). Global Warming Central: Debate number three. Http://www.law.pace. edu.

Campana, Á. (2017). La inversión privada en el sector educación: un análisis de las APP y $\mathrm{OxI}$ en infraestructura educativa. Grupo propuesta ciudadana. 
Chon Yamasato, E. (2006). Sub Sistema de Seguimiento, Análisis y Evaluación de Planes de Mediano Plazo: documento de trabajo. Repositorio del Minedu.

Cisneros-Méndez, M. (2017). La problemática de las asociaciones público privadas en el Perú con ocasión de la cláusula anticorrupción. Repositorio de la Universidad de Lima.

Eco Finanzas (2009). La teoría poblacional de Thomas Malthus. Recuperado el 20 de agosto de 2013. Disponible en http://www. eco-finanzas.com/economia/economistas/ Thomas-Malthus-teoria-poblacional.htm.

Fiestas, S. R. (2010). Priorización de los proyectos de inversión pública mediante el análisis de componentes principales. Repositorio de la UNI.

George, C.M., Sima, L., Jahuira Arias, H., Mihalic, J., Cabrera, L., Danz, D., Checkley, W. y Gilman, R. (2014). Exposición al arsénico en el agua potable: una gran amenaza inadvertida para la salud en Perú. Boletín de la Organización Mundial de la Salud, 92(8), 545-620. Recuperado de http://www.who.int/ bulletin/volumes/92/8/13-128496-ab/es/>.

Jaramillo S., Arboleda K. y Guerra J. (2010). Causas y consecuencias de la superpoblación. Recuperado el 20 deagosto de 2013. Disponible en: http://lasobrepoblacion2010.blogspot. mx/2010/09/causas-y-consecuencias-dela_7028.html.

Marín, M. y Medina, F. J. (1995). Actitudes hacia el medio ambiente y conducta ecológica responsable. En E. Garrido y C.

Herrero (Comps.), Psicología Política, Jurídica y Ambiental, 345-355. Salamanca, España.: Eudema. Odum, E. P. (1992). Ecología: bases científicas para un nuevo paradigma. Barcelona, España.: Vedrá. (Edicción original: Sinauer Associates, Inc, Publishers, 1989).

Ortegón, E. (2005). Metodología del marco lógico para la planificación, el seguimiento y la evaluación de proyectos y programas, 42. United Nations Publications.

Sánchez, J. (2008). "El crecimiento de la población mundial", Editorial Tirant Lo Blanch, S.L, 1 ra edición, español, (653 pág.).

Seligman, C. (1989). Environmental ethics. Journal of Social Issues, 45(1), 169184.

Suárez, E.; Hess, S. y Martínez-Torvisco, J. (1992). Hacia un modelo de la conducta ecológica responsable y de la participación ambiental. En M. Amérigo, J.I., Aragonés y J.A. Corraliza (Comps.), El comportamiento en el medio natural y construido, 113-119. Orellana. Badajoz, España.: Junta de Extremadura.

Thompson, S. C. y Stoutemyer, K. (1991). Water use as a commons dilemma. The effects of education that focuses on logterm consequences and individual action. Environment and Behavior, 23(3), 314-333.

Tracy, A. P. y Oskamp, S. (1984). Relationships among ecologically responsible behaviors. Journal of Environmental Systems, 13, 115-126.

Wilson, Edward O. (1978). Ecología, evolución y biología de poblaciones. Ediciones Omega. ISBN 978-84-282-0502-3.

Zegarra Quevedo, J. M. (2009). Sistema nacional de inversión pública. Repositorio de la UNI. 